\title{
Mural INTERNACIONAL
}

Mural Internacional é a revista eletrônica semestral do Programa de Pós-Graduação em Relações Internacionais da Universidade do Estado do Rio de Janeiro. Seu objetivo é debater temas relevantes das Relações Internacionais como a política internacional, políticas externas, economia política internacional, processos de integração regional, instituições internacionais, processos migratórios internacionais, relações culturais internacionais, discussões teóricas e/ou metodológicas e temas da atualidade de determinadas regiões.

As opiniões expressas nos artigos são de responsabilidade de seus respectivos autores.

Seu download é gratuito, a partir do site http://www.e-publicacoes.uerj.br/index.php/muralinternacional

Editora-chefe:

Tatiana Oliveira (PPGRI/UERJ)

Assistente de Editoração:

Cristiane Ferreira Baptista

Revisão e Webdesign:

Cristiane Ferreira Baptista

Editores associados

Danielle Costa da Silva (PPGRI/UERJ)

Lorena Granja (PPGRI/UERJ)

Maurício Santoro Rocha (PPGRI/UERJ)

\section{Conselho Editorial}

Alejandro Simonoff (Universidad de La Plata)

Alexis Toríbio Dantas (PPGRI/UERJ)

Ana Paula Balthazar Tostes (PPGRI/UERJ)

Andrés Malamud (Universidade de Lisboa)

Anna Ayuso (CIDOB/Barcelona)

Antônio Carlos Lessa (Universidade de Brasília)

Bruno de Moura Borges (PPGRI/UERJ)

Cláudio de Carvalho Silveira (PPGRI/UERJ)

Clóvis Eugenio Georges Brigagão (Universidade Cândido Mendes)

Erica Simone Almeida Resende (IUPERJ/UCAM)
Fernando Roberto de Freitas Almeida

(Universidade Federal Fluminense)

Franklin Trein (Universidade Federal do Rio de Janeiro)

Gelson Fonseca Júnior (Ministério de Relações

Exteriores)

Gladys Teresita Lechini (Universidad Nacional de Rosario)

Hugo Rogelio Suppo (PPGRI/UERJ)

Javier Vadell (Pontifícia Universidade Católica de Minas Gerais)

José Briceño Ruiz (Universidad de Los Andes/Venezuela)

Layla Dawood (PPGRI/UERJ)

Lená Medeiros de Menezes (PPGRI/UERJ)

Letícia Pinheiro (IESP/Universidade do Estado do Rio de Janeiro)

Lia Cecília Baker Fonseca Valls Pereira (PPGRI/UERJ)

Marcelo de Almeida Medeiros

(Universidade Federal de Pernambuco)

Marcelo Valença (PPGRI/UERJ)

Maria Regina Soares de Lima

(IESP/Universidade do Estado do Rio de Janeiro)

Miriam Gomes Saraiva (PPGRI/UERJ)

Mônica Lessa (PPGRI/UERJ)

Paulo Afonso MonteiroVelasco (PPGRI/UERJ)

Tullo Vigevani

(Universidade Estadual Paulista Júlio de Mesquita Filho)

\section{Correspondência:}

Realização:

Programa de Pós-Graduação em Relações Internacionais

Instituto de Filosofia e Ciências Sociais/Universidade do Estado do Rio de Janeiro

Rua São Francisco Xavier, 524, $9^{\circ}$ Andar, bloco F, sala 9037.

Rio de Janeiro RJ | Cep.22071-030 Brasil

Tel. (55) (21) 2334-0678 | E-mail: ppgri.revista@ gmail.com

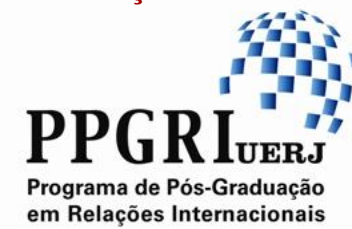




\section{CONTRIBUIÇÃO ESPECIAL | SPECIAL CONTRIBUTION}

Embaixador Sérgio Tutikian: um pioneiro no Oriente Médio |Ambassador Sérgio Tutikian: a pioneer in the Middle East MONIQUE SOCHACZEWSKI GOLDFELD

101-116

\section{ARTIGOS | ARTICLES}

As relações de paz e guerra entre Peru e Equador no contexto do complexo de segurança regional | The relations of peace and war between Peru and Ecuador in the context of regional security complex SAMUEL CORREA DUARTE

117-138

Extensão universitária em Relações Internacionais: ditadura e transição à democracia na América do Sul |Science outreach in the field of International Relations: dictatorship and transitional justice in South America

CLARISSA FRANZOI DRI, ANDRESSA CAROLINE MOLINARI DA SILVA \& MARINA LAZAROTTO DE ANDRADE

139-150

Política externa e regionalismo em um ambiente internacional em transformação | Foreign policy and regionalism in a changing international system

HAROLDO RAMANZINI JUNIOR

151-166

Processo Decisório e Política Externa nos Pequenos Estados da Periferia: Cabo Verde e GuinéBissau | Decision-Making Process and Foreign Policy in Small Peripheral States: Cape Verde and Guinea-Bissau

TIMÓTEO SABA M'BYNDÉ

167-185

Geopolítica como Luta de Classes: Marxismo Político, Relações Internacionais e Sociologia Histórica | Geopolitics as Class Struggle: Political Marxism, International Relations and Historical Sociology

PEDRO DUTRA SALGADO

186-202

A Política Externa Brasileira como Política Pública: a proposta de um Conselho Nacional e a experiência do Comitê Brasileiro de Direitos Humanos e Política Externa|Brazilian foreign policy as public policy: the proposal of a National Council and the experience of the Human Rights and Foreign Affairs Brazilian Committee

DANIELLE COSTA DA SILVA \& MAGNO KLEIN

203-218

O Brasil na promoção da democracia: redemocratização e política externa no pós-1985 | The Brazilian agenda of democracy promotion: redemocratization and foreign policy after 1985 GUILHERME FERREIRA SORGINE 219-233

"The United States And its Allies are on the Right Side of History": a Guerra Global ao Terror e suas representações | "The United States And its Allies are on the Right Side of History": the Global War on Terror and its representations MARCELO MELLO VALENÇA \& YESA PORTELA ORMOND 234-247 


\section{SUMÁRIO | TABLE OF CONTENTS}

\section{RESENHAS | BOOK REVIEWS}

Bauman, Zygmunt. Estranhos à Nossa Porta. Rio de Janeiro: Zahar, 2017, 117p. ISBN 978-85-3781610-3

CAMILA DE' CARLI

248-251

Daróz, Carlos. O Brasil na Primeira Guerra Mundial: a longa travessia. São Paulo: Editora Contexto, 2016, 208 p. ISBN: 978-85-7244-952-6

TOMAZ ESPÓSITO NETO

252-254

NOMINATA | REVIWERS ACKNOWLEDGEMENTS

255 